Some people do not present AMS on several altitude exposures, however, unexplainably, they may have it occasionally. Several tests have been tried in order to determine who will have AMS, however, none to date are able to achieve with absolute certainty who will get AMS. Some tests include having subjects breathe a hypoxic mixture. Others have created exercise protocols with exposure to hypoxia and some other techniques. However, none have been successful. We have successfully diagnosed and treated patients with AMS of different intensity, over many years of work at high altitude, some having undiscovered pathologies that became evident on exposure to high altitude. For example, interauricular communications, nephropaties, leading to essential hypertension, cardiac arrhythmias, presence of a kidney stone that did not block the urine pathway completely, abnormal arteries in the kidney, sequelae of pulmonary disease with localized fibrosis, relative anemia due to poor bone marrow response, falciform anemia, and many others. However, those that apparently have no evident disease can present with severe cases of dehydration, particularly while doing exercise at high altitude. This of course, can be quite debilitating and if pushed beyond rationale can lead to severe complications. Performing a very precise diagnosis is difficult and there is still much to study in order to understand hypoxia thoroughly. One of the explanations for the occasional presence of AMS could be the presence of subclinical viral infections. We live with viruses, and when the virus is not so aggressive and not present in sufficient quantities, the organism has an internal struggle that uses up the energy. If the person ascends a mountain in this condition, his reserve to face hypoxic stress is limited and hence complications may appear. Likewise, if the person prior to the ascent had an different type of food, with unusual spices, or with unusual bacteria, then there is an inflammation of the bowels and the endothelial cells of the gastrointestinal tract, particularly at the colon level, may not be able to absorb water as efficiently as they would under normal circumstances. This could lead to a more severe case of dehydration and hence AMS. There are probably many other factors which include stress from other psychological difficulties, or even exposure to toxics that are inhaled and produce inflammation in the respiratory tract. Furthermore, those training at high altitude can overdo the exercise efforts, with great willpower, leading to very increased pulmonary artery pressure and hence a dilated right heart. This overcharged effort of the muscle cells in the right heart can give rise to inflammation and microlesions that render the heart mildly insufficient in its capacity for pumping blood. On going higher in a sports competition, the optimal heart function is diminished and hence AMS can appear. This is due to the fact that the Adaptation to High Altitude Formula has not been respected in relation to time, where an adequate and timely increase of red blood cells is essential thereby generating subacute high altitude heart disease.

\section{Low Altitude Peripheral Edema (LAPE): The Oppo- site of HAPE or HACE}

Gustavo Zubieta-Calleja, MD; Natalia Zubieta-DeUrioste

High Altitude Pulmonary and Pathology Institute (IPPA), La Paz, Bolivia

Millions of permanent high-altitude residents, born at high altitude, living normal lives, practicing sports, sleeping well, reproducing, and enjoying entertainment, occasionally descend to sea level, for work or leisure. This is a change where the organism perfectly adapted to chronic hypoxia is suddenly exposed to a hyperoxic environment and needs to adapt to the new circumstance. Although no alarming symptoms and signs such as those that can be seen in acute mountain sickness exist like headaches, nausea, or vomiting, there are signs that show evident changes in the body. One of the most striking is edema of lower limbs that can become more pronounced at 2 weeks of stay. Although there may be an initial edema due to long airplane travel times, this usually goes away within one or two days. However, after around 2 weeks, a positive Godet sign develops in both legs that can be quite impressive. The Godet sign, found by pressing during a few seconds on the skin in front of the tibia, displaces excessive fluid found in the interstitial subcutaneous spaces and gives rise to the formation of an evident concave impression. This sign is usually found in patients suffering from cardiac insufficiency, renal insufficiency, anasarca with low blood protein levels, or inflammation. If a sea level physician would evaluate one of the high-altitude residents, at that moment, he would surely think of cardiac insufficiency, and may event start treatment with diuretics or digitalis. Possible mechanisms involve an increase of sialic acid, found above or borderline of the normal maximum limits found during tests, in 4 subjects. Urine tests also showed an acid $\mathrm{pH}=6.0$, in spite of vegetarian diets, on a recent trip to India. Upon ascent to high altitude, there is central edema and that is why acute mountain sickness, high altitude pulmonary edema and high altitude cerebral edema occur. Conversely, on descending to sea level, peripheral edema occurs. Going higher, oxygen needs to be transported preferably to the life-sustaining organs: brain, heart, and lungs, whereas going lower there is "excessive" amounts of oxygen and peripheral edema occurs possibly as a defense mechanism to reduce oxygen transport to the life sustaining organs, as it is sensed toxic. The hematologic adaptation with a decrease of the hematocrit to sea level values, is linear and takes around 20 days, going from $3500 \mathrm{~m}$. Hence, once the hematocrit is at the optimal values for sea level, the peripheral edema decreases and is not as evident. Consequently, the Altitude Adaptation Formula stands valid even going down and hence it is best to include the altitude change in the denominator:

Adaptation to altitude $=$ time/altitude $\Delta$

\section{Rebuilding of Carbohydrate and Lipid Metabolism Under Hypoxia: Regularities and Therapeutic Possibilities}

A. Portnychenko, MD, PhD DrSc; V. Portnichenko, MD, PhD;

T. Lapikova-Bryhinska; V. Nosar, PhD; M. Vasylenko, PhD;

H. Portnichenko

International Centre for Astronomical, Medical and Ecological

Research, NAS of Ukraine; Bogomoletz Institute of Physiology, NAS of Ukraine; 4 Bogomoletz str., 01024 Kyiv, Ukraine

Previously, we have found that the effects of chronic (staying in midlands) or moderate periodic hypoxia as well as hypoxic preconditioning is accompanied by beneficial rebuilding in lipid and carbohydrate metabolism in human and animals: a reduction of total cholesterol and its fractions in blood plasma, a hypoglycemic reaction or normalization of elevated blood glucose, and a decrease of impaired glucose tolerance. This metabolic rebuilding has a phase character during the adaptation to hypoxia and following deadaptation. Changes in metabolism during hypoxia occur in a consecutive manner and mediate by induction and repression of target genes following activation of hypoxia responsible signal ways and transcription factors. In particular, we showed the alternate transactivation of HIF-1alpha and -3alpha subunits, and the restructuring of glucose transport by consecutive induction of GLUT-4 and -1 in rat myocardium and lungs. Mitochondrial respiration is consequentially shifted from down - to upregulation, and from carbohydrate to mainly lipid substrate using. In human, we found attenuation of transcriptional regulator of cholesterol synthesis SREBP1, leptin and insulin-like growth factor IGF-1 levels in blood plasma under prolonged hypoxic conditions, as well as positive correlation with HIF-dependent protein IGF-1 and HDL-cholesterol level. In middle-aged people with metabolic disorders (metabolic syndrome, diabetes mellitus, dyslipidemia), a favorable influence of staying in midlands, moderate acute or periodic hypoxia was found, but metabolic pathology was associated with altered patterns of regulatory protein expression. The data suggest the close relationship between carbohydrate and lipid metabolism under hypoxia, and evidence the possibility of hypoxic correction of metabolic disorders as diabetes, dyslipidemia and metabolic syndrome based on phase processes of metabolic rebuilding.

\section{Modification and Further Applications of the Adap- tation to High Altitude Formula}

Gustavo Zubieta-Calleja, MD; Natalia Zubieta-DeUrioste

High Altitude Pulmonary and Pathology Institute (IPPA), La Paz, Bolivia

Adaptation to high altitude is fundamental for life to go on. It is a time and altitude dependent phenomena, because the organism needs the adequate time to build the defense mechanisms to face environmental changes. Quoting the late Gustavo Zubieta-Castillo: "The organic systems of human beings and all other species tend to adapt to any environmental change and circumstance within an optimal period of time, and never tend towards regression which would inevitably lead to death."

Adaptation to altitude $=$ time/altitude $\Delta$

It is important to note that altitude changes require adaptation going both ways: going up in altitude or for high altitude residents, going down. This formula was originally developed using the changes in altitude going higher, because it was thought worldwide that the normal physiology of the body was developed at sea level, only. However, high altitude residents are perfectly adapted to life in their own environment. Hence it has been found convenient to include in the formula, in the denominator "altitude $\Delta$ " i.e. altitude change, instead of only the term 
"altitude." This we find pivotal because the organism of high altitude residents going down to sea level, has to adapt to the "relative hyperoxic environment." It is wrongly assumed that for high altitude residents, going to a lower altitude where there is more oxygen pressure, as a result of a higher barometric pressure, is beneficial. We strongly question this, as high altitude residents, being born, developed, and carrying out normal lives in the mountain cities of the world living in perfect harmony with our environment. We, as Andean high altitude residents, have higher hematocrit and hemoglobin values, as the fundamental compensating biological response. All living beings, humans, animals and plants and presumably other microscopic organisms suffer biologic changes on barometric pressure changes. In other words, physical changes in the environment, induce biological changes. The rules governing physics are imbedded within biology.

The formula of adaptation is not only useful for high altitude adaptation, but rather can be used for any type of adaptation, where the organism in order to survive, needs to find the most energy efficient, fastest rebuild or healing process of the organic systems. An example can be found with a wound in the skin. When the two borders are sutured, the healing process takes around 1 week. This would be: healing adaptation $=$ time/tissue

This formula changes in time if the tissue is skin or bone, the later requiring a longer time of a few months. This formula also varies with age, taking longer for the older people:

\section{Adaptation to healing $=$ time/age}

It should be well understood, that the healthier the subject, the better the adaptation. But it also applies to allergic reactions to mosquito bites in those not habituated to them in comparison to those living in those areas. Likewise, a viral aggression like flu, has a latent period of immunity that follows the same rule: Adaptation to viral aggression $=$ time/type of viral agent.

This formula has other additional variables like nutrition, stress and aggressiveness of the viral agent. Furthermore it also applies to adaptation to smell. When a new perfume is smelled for the first time it is very strong, but persistent use of it changes the capacity of sensing it:

Adaptation to smell $=$ time/scent.

Muscle adaptation to exercise also follows the same formula:

Adaptation to exercise $=$ time/exercise $\Delta$.

The more exercise, the greater the hypertrophy of the muscles and vice-versa.

In conclusion the Adaptation Formula is a fundamental formula that applies to all biological adaptation processes in the multiple survival mechanisms of all living beings, and can be generalized as:

Adaptation $=$ time $/$ change

\section{Neural Mechanisms Involved in Autonomic and Respiratory Changes in Rats, Submitted to Short- Term Sustained Hypoxia}

Daniela Accorsi-Mendonça, PhD; Carlos E.L. Almado, PhD; Leni G.H. Bonagamba, BS; Kauê M. Costa, MS; Jaci A. Castania, BS; João Henrique Costa-Silva, PhD; Daniel B. Zoccal, PhD; Davi J.A. Moraes, PhD; Benedito H. Machado (presenter), PhD Department of Physiology, School of Medicine of Ribeirão Preto, University of São Paulo, 10409-900, Ribeirão Preto, SP, Brazil

Humans ascending to high altitudes are submitted to sustained hypoxia (SH), activating peripheral chemoreflex with several autonomic and respiratory responses. We analyzed the effect of short-term $\mathrm{SH}$ (24 hours, $\left.\mathrm{F}_{\mathrm{I}} \mathrm{O}_{2} \quad 10 \%\right)$ on the cardiovascular parameters in non-anesthetized rats and on the processing of cardiovascular and respiratory reflexes using in situ and in vitro preparations. SH produced hypertension in awake rats and increased the basal sympathetic activity in an in situ preparation and these effects may be related to changes in respiratorysympathetic coupling. SH increased both the sympatho-inhibitory and bradycardiac components of baroreflex and the sympathetic and respiratory responses of peripheral chemoreflex. Electrophysiological properties and synaptic transmission in the nucleus tractus solitarius (NTS) neurons, the first synaptic station of afferents of baro- and chemoreflex, were also evaluated using brainstem slices (in vitro studies). The second-order NTS neurons were identified by previous application of fluorescent tracer onto carotid body for chemoreceptor afferents or onto aortic depressor nerve for baroreceptor afferents. SH increased the intrinsic excitability of NTS neurons. Delayed excitation, caused by A-type potassium current $\left(\mathrm{IK}_{\mathrm{A}}\right)$ was observed in most of NTS neurons from control rats. The $\mathrm{IK}_{\mathrm{A}}$ amplitude was higher in identified second-order NTS neurons from control than in
$\mathrm{SH}$ rats. $\mathrm{SH}$ also blunted the astrocytic inhibition of $\mathrm{IK}_{\mathrm{A}}$ in NTS neurons and increased the synaptic transmission in response to afferent fibers stimulation. The reduction of glial cell density was also observed after $\mathrm{SH}$ protocol. Therefore, short-term SH produces changes in structural and functional integrity of glial cells, which are essential for neuronal activity and may contribute to the increase in cardiovascular reflex sensitivity (baro- and chemoreflex) and the development of hypertension in awake rats. Financial support: FAPESP, CAPES and $\mathrm{CNPq}$.

\section{Prospective Validation of Framingham Criteria for Congestive Heart Failure Diagnosis in a High-Altitude Population}

\author{
Z. Sovero ${ }^{2}$; E. Capcha ${ }^{2}$; W. Calderón-Gerstein, $\mathrm{MD}^{1,2}$
}

${ }^{1}$ Hospital Nacional Ramiro Prialé Prialé, EsSalud, Huancayo, Junín, Perú, ${ }^{2}$ Universidad Nacional del Centro del Perú, Huancayo, Perú

Purpose.-To determine the validity of Framingham criteria for heart failure diagnosis in a high-altitude population.

Material and Methods.-This is a prospective, observational, descriptive, and transversal study. The study population was comprised of 97 inpatients hospitalized at Internal Medicine Service at Hospital Nacional del Centro EsSalud Huancayo, located at an altitude of $3240 \mathrm{~m}$ above sea level. Patients included presented with dyspnea on exertion or at least one major Framingham criterion. Echocardiograms were performed with a Sonos 1000 echocardiographer, with color Doppler, B-mode and M-mode techniques. Dichotomous variables were analyzed by Mantel - Haenszel chi square test. A p $<0.05$ was considered significant.

Results.-Most patients were male (51.6\%). Subject age was $69.5 \pm[\mathrm{SD}$ 14.647] (20-90) years (mean \pm SD with range). A total of $73.2 \%$ of patients were elderly $(\mathrm{n}=71)$. The most frequent major criteria found were: cardiomegaly $(88.9 \%)$, hepatojugular reflux $(79.2 \%)$ and jugular engorgement $(69.4 \%)$. The most important minor criteria were: exertional dyspnea $(91.7 \%)$, pleural effusion $(69.4 \%)$ and lower limb edema (58.3\%). Framingham diagnostic criteria were present in $98.6 \%$ of patients with heart failure but had a specificity of only $12 \%$. The specificity rose to $76 \%$ by using 4 major criteria and to $100 \%$ when using 5 criteria. Unfortunately, the sensitivity fell at the same time to $62.5 \%$ and $31.9 \%$, respectively.

Conclusions.-Framingham criteria are highly sensitive but nonspecific for the diagnosis of heart failure in high-altitude populations.

\section{Development and Validation of New Sepsis Criteria at High-Altitude}

Walter Calderón-Gerstein, $\mathrm{MD}^{1,2}$

${ }^{1}$ Hospital Nacional Ramiro Prialé Prialé, EsSalud, Huancayo, Junín, Perú, ${ }^{2}$ Universidad Nacional del Centro del Perú, Huancayo, Perú

Introduction.-Current sepsis diagnostic criteria lose precision at high altitude due to physiological variation in vital functions. Because of that, it is necessary to elaborate new sepsis diagnostic criteria, especially designed for its use at populations living at $2000 \mathrm{~m}$ above sea level or higher altitudes.

Methods.-This is a prospective, longitudinal and observational study, that took place at EsSalud Regional Hospital at Huancayo. Two cohorts were studied: a derivation cohort (394 patients) and a validation cohort (123 patients). A total of 147 adult patients with sepsis diagnosis and 247 healthy controls were included in the derivation cohort. Sepsis was confirmed with cultures, and diagnosis of infection was made according to CDC and McGeer criteria, in patients admitted to Intensive and Intermediate Care Unit from January 2007 to April 2008. Each case was followed up to discharge or death. With the obtained data, new diagnostic models were devised. A validation cohort of 72 patients with sepsis, 51 hospitalized patients without sepsis and 120 nonhospitalized normal subjects was developed (validation cohort) with patients admitted to Internal Medicina Seervice from January 2012 to December 2013. Diagnostic criteria were evaluated with $2 \times 2$ tables, and specificity, sensitivity, negative and positive predictive values, as well as ROC AUC values were determined. Relative risk values and $95 \%$ confidence intervals are presented.

Results.-A total of 10 physiological variables were obtained to develop the new criteria. Three of them were selected: respiratory rate $>23$, temperature $>37.8^{\circ} \mathrm{C}$ or $37.5^{\circ} \mathrm{C}$, and heart rate $>85$ beats/minute. More specific alternative 